\title{
Prevalência da síndrome dos ovários policísticos em uma instituição de ensino superior da cidade de Cajazeiras - PB
}

\author{
Prevalence of polycystic ovary syndrome in a higher education institution in the city of Cajazeiras -
} PB

\author{
Prevalencia del síndrome de ovario poliquístico en una institución de educación superior de la \\ ciudad de Cajazeiras - PB
}

Recebido: 29/03/2021 | Revisado: 08/04/2021 | Aceito: 12/04/2021 | Publicado: 24/04/2021

Emanoely Gomes dos Anjos ORCID: https://orcid.org/0000-0003-4799-3970 Faculdade Santa Maria, Brasil

E-mail: gomesemanoely@gmail.com

Graziela Nogueira Eduardo

ORCID: https://orcid.org/0000-0001-9942-9496 Faculdade Santa Maria, Brasil E-mail: 20172003004@fsmead.com.br

Karla Brehnda Cabral Liberato Figueiredo ORCID: https://orcid.org/0000-0002-0681-8239 Faculdade Santa Maria, Brasil

E-mail: brehndacliberato@gmail.com

Samara Alves Brito

ORCID: https://orcid.org/0000-0003-0629-7936 Faculdade Santa Maria, Brasil

E-mail:000604@fsmead.com.br

Gislayne Tacyana Santos Lucena

ORCID: https://orcid.org/0000-0003-1833-9208 Faculdade Santa Maria, Brasil

E-mail: gislayne.lucena@hotmail.com

\begin{abstract}
Resumo
O objetivo deste trabalho foi realizar um levantamento da prevalência de casos da SOP entre discentes da Faculdade Santa Maria, da cidade de Cajazeiras, Paraíba, analisando o método diagnóstico, medidas terapêuticas empregadas e o caráter genético atribuído à síndrome. O presente estudo foi desenvolvido de acordo com o delineamento de uma pesquisa quantitativa do tipo transversal observacional descritiva. Foram selecionadas 50 acadêmicas de forma não probabilística intencional. Os dados foram coletados através da aplicação de um questionário contendo dez perguntas. A análise dos dados revelou uma porcentagem de $24 \%$ das entrevistadas com o distúrbio, além disso, o método diagnóstico mais utilizado em tais casos foi ultrassonografia da pelve, citado por $46,2 \%$ das mulheres com SOP, seguido do exame clínico, representado pelas alterações no ciclo menstrual, que foi relatado por 30,8\% destas participantes. Todas as entrevistadas com a síndrome relataram fazer algum tipo de tratamento e a medida terapêutica mais empregada foi o uso de anticoncepcionais orais, seguido da adoção de um estilo de vida saudável. Nenhuma das entrevistadas fazia uso de medicamentos sensibilizantes à insulina. O sintoma clínico mais comum entre estas mulheres foi a alteração do ciclo menstrual, presente em 91,6\% delas. A média de idade entre as mulheres com SOP foi de 20,50 anos. Proporcionalmente, mais mulheres que relataram ter familiares com SOP tinham SOP. Tal dado sugere a condição genética envolvida na etiopatogenia da mesma, que também pode ser influenciada por fatores ambientais.
\end{abstract}

Palavras-chave: Síndrome do ovário policístico; Hiperandrogenismo; Sistema reprodutor feminino.

\begin{abstract}
The objective of this study was to evaluate the prevalence of PCOS cases among students of the Faculty of Santa Maria, Cajazeiras, Paraíba, analyzing the diagnostic method, therapeutic measures used and the genetic character attributed to the syndrome. The present study was developed according to the design of a descriptive observational cross-sectional quantitative study. We selected 50 academics in a non-probabilistic way. The data were collected through the application of a questionnaire containing ten questions. The analysis of the data revealed a percentage of $24 \%$ of those interviewed with the disorder. In addition, the most used diagnostic method in such cases was pelvic ultrasonography, cited by $46.2 \%$ of women with PCOS, followed by clinical examination, represented by the changes in the menstrual cycle, which was reported by $30.8 \%$ of these participants. All the interviewees with the syndrome reported doing some type of treatment and the most used therapeutic measure was the use of oral contraceptives, followed by the adoption of a healthy lifestyle. None of the women interviewed used insulin-sensitizing drugs. The
\end{abstract}


most common clinical symptom among these women was the alteration of the menstrual cycle, present in $91.6 \%$ of them. Proportionately, more women who reported having relatives with PCOS had PCOS. Such data suggest the genetic condition involved in the etiopathogenesis of the same, which can also be influenced by environmental factors.

Keywords: Polycystic ovarian syndrome; Hyperandrogenism; Female reproductive system.

\section{Resumen}

El objetivo de este estudio fue evaluar la prevalencia de casos de SOP entre estudiantes de la Facultad de Santa María, Cajazeiras, Paraíba, analizando el método diagnóstico, las medidas terapéuticas utilizadas y el carácter genético atribuido al síndrome. El presente estudio se desarrolló de acuerdo con el diseño de un estudio cuantitativo transversal observacional descriptivo. Seleccionamos 50 académicos de forma no probabilística. Los datos fueron recolectados mediante la aplicación de un cuestionario que contenía diez preguntas. El análisis de los datos reveló un porcentaje del $24 \%$ de los entrevistados con el trastorno. Además, el método diagnóstico más utilizado en estos casos fue la ecografía pélvica, citada por el $46,2 \%$ de las mujeres con SOP, seguida del examen clínico, representado por los cambios en el ciclo menstrual, que fue reportado por el 30,8\% de estas participantes. Todos los entrevistados con el síndrome refirieron realizar algún tipo de tratamiento y la medida terapéutica más utilizada fue el uso de anticonceptivos orales, seguida de la adopción de un estilo de vida saludable. Ninguna de las mujeres entrevistadas utilizó fármacos sensibilizantes a la insulina. El síntoma clínico más común entre estas mujeres fue la alteración del ciclo menstrual, presente en el 91,6\% de ellas. Proporcionalmente, más mujeres que informaron tener parientes con SOP tenían SOP. Dichos datos sugieren la condición genética involucrada en la etiopatogenia de la misma, que también puede estar influenciada por factores ambientales.

Palabras clave: Síndrome de ovario poliquístico; Hiperandrogenismo; Sistema reproductivo femenino.

\section{Introdução}

Descrita pela primeira vez pelos ginecologistas americanos Irving Freiler Stein e Michael Leventhal no ano de 1935 e nomeada como Síndrome de Stein-Leventhal, a síndrome dos ovários policísticos (SOP), que passou a assim ser conhecida a partir da década de 1960, caracterizou-se a princípio por quadros de amenorreia, associados à obesidade, hirsutismo e ovários com volume anormal (Facio-Lince García et al., 2015).

As manifestações clínicas da SOP geralmente surgem logo após a menarca, durante o período da adolescência, onde muitas vezes ocorrem modificações na vida das mulheres, como ganho de peso e sedentarismo (Baptista; Vieira; Meireles, 2016; Silva; Pardini; Kater, 2006). Sua etiologia ainda não é totalmente descrita, contudo, várias hipóteses já foram levantadas. Alterações na produção de gonadotrofinas (GnRH), liberação hipofisária dos hormônios luteinizantes (LH) e folículo estimulante (FSH), nas funções ovarianas e suprarrenal, resistência insulínica (RI) e condições genéticas se apresentam como os principais fatores para o desencadeamento da doença. Além disso, antecedentes de baixo peso ao nascer, e puberdade precoce, proporcionam risco aumentado para o desenvolvimento da síndrome. Deste modo, a origem da SOP não se configura em um distúrbio endócrino específico, mas em alterações complexas, nas quais, envolvem-se diversas questões genéticas, hormonais e condições ambientais (Rojas, 2018).

Devido à variedade das características clínicas da SOP, alguns consensos foram realizados ao longo dos anos em busca de um método diagnóstico para a síndrome. Os mesmos puderam definir que se faz necessário a constatação de no mínimo dois, dos seguintes critérios: anovulação crônica; sinais clínicos e/ou laboratoriais de hiperandrogenismo e presença de ovários com múltiplos cistos ao exame ultrassonográfico. Além disso, é fundamental a exclusão de outras causas de hiperandrogenismo, a exemplo da síndrome de Cushing, deficiência enzimática da suprarrenal, hiperprolactinemia, e distúrbios da glândula tireoide (Ávila et al., 2014).

O tratamento da SOP é direcionado principalmente aos sinais clínicos do hiperandrogenismo, regulação dos ciclos menstruais e contenção dos riscos metabólicos. Além disso, a escolha do método terapêutico irá depender da gravidade dos sintomas e os objetivos particulares da mulher. Diante mão, a adoção de um estilo de vida saudável, através da introdução de bons hábitos alimentares e atividades físicas regulares se apresentam como a primeira linha de tratamento, especificamente para aquelas que apresentam sobrepeso. Para este grupo específico bem como às que apresentam resistência insulínica 
independente do peso, os medicamentos sensibilizadores da insulina auxiliam na melhora desta condição e ainda ajudam na recuperação da capacidade ovulatória, para este fim, o fármaco mais utilizado e discutido é a metformina (MTF). A terapia com anticoncepcional oral apresenta grande eficácia na regulação da menstruação, mas não se aplica àquelas que desejam engravidar. Por este motivo os medicamentos indutores de ovulação, como o citrato de clomifeno também são utilizados em mulheres com a síndrome. Os tratamentos estéticos também são úteis e ajudam a amenizar a expressão física do excesso de andrógenos, como o hirsutismo, alopecia e acne (Moura et al., 2011; Kogure; Reis, 2017).

Estima-se que a SOP acometa cerca de 5 a $10 \%$ da população mundial feminina em idade fértil, caracterizando-se como uma das disfunções endócrino-ginecológicas mais frequentes. Mundialmente, aproximadamente 105 milhões de mulheres com idade entre 15 e 49 anos são portadoras da doença. A síndrome ainda é considerada uma das principais causas de hiperandrogenismo nas mulheres, ademais, em cerca de $72 \%$ a $82 \%$ dos casos de SOP é observado o aumento de andrógenos circulantes (Rehme, et al., 2013a; Silva; Pardini; Kater, 2006).

A longo prazo, a SOP pode ser responsável pelo desenvolvimento de problemas como infertilidade anovulatória, diabetes mellitus do tipo 2, alterações metabólicas e cardiovasculares, hiperplasia e neoplasia endometrial e até mesmo distúrbios psicológicos, problemas de autoestima e sociais (Ávila et al., 2014; Spritzer; Morsch; Wiltgen, 2005). Deste modo, tal síndrome representa um risco à saúde e qualidade de vida da mulher em idade reprodutiva, e investigar sua prevalência na comunidade em que estamos inseridos é importante para que possamos alertar as pessoas acerca da doença e prevenir danos maiores ao bem-estar das portadoras. A literatura ainda apresenta carência de pesquisas epidemiológicas acerca da SOP, fato que atribui considerada relevância ao presente estudo por abordar esta temática pouco explorada. O objetivo deste trabalho foi realizar um levantamento da prevalência de casos da síndrome dos ovários policísticos entre discentes da Faculdade Santa Maria, da cidade de Cajazeiras, Paraíba, analisando o método diagnóstico, medidas terapêuticas empregadas e o caráter genético atribuído à síndrome.

\section{Metodologia}

Trata-se de uma pesquisa quantitativa do tipo transversal observacional descritiva. Os indivíduos foram selecionados de forma não probabilística intencional. A população foi formada por discentes matriculadas na instituição de ensino superior privada Faculdade Santa Maria - FSM, localizada na Rodovia BR 230, km 504, bairro Cristo Rei da cidade de Cajazeiras, estado da Paraíba, Brasil.

A amostragem foi constituída pelas primeiras cinquenta voluntárias que aceitaram fazer parte da pesquisa. Os indivíduos selecionados como participantes da pesquisa foram aconselhados pela pesquisadora e sua equipe para assinatura do Termo de Consentimento Livre e Esclarecido - TCLE, impresso em duas vias, uma para o pesquisado e outra para o pesquisador, no qual estão descritos os objetivos, benefícios, riscos e metodologia a ser aplicada na realização do trabalho. Após aceitação dos mesmos, iniciou a coleta de dados a partir da aplicação de questionário contendo 10 questões subjetivas e objetivas com perguntas de resposta do tipo binária e de múltipla escolha, o qual foi aplicado uma única vez e entregue em anexo com o TCLE. A coleta dos dados foi realizada no mês de outubro do ano de 2018. O preenchimento dos documentos está livre de rasuras, assegurando assim, fidedignidade no resultado da pesquisa. Todo o material utilizado foi fornecido pela equipe de pesquisadores.

As informações coletadas estão sob sigilo ético profissional e arquivadas na secretaria acadêmica da Faculdade Santa Maria - FSM. O instrumento de coleta de dados será eliminado como documentos caracterizados do tipo de guarda temporária depois de decorrido dois anos a contar da data do termino da pesquisa. 
Foi preconizado que as participantes apresentarem idade entre 15 e 49 anos, intervalo de tempo que, segundo a OMS (2011) representa os anos de fertilidade feminina. A participante será excluída do estudo caso constatada fraude, omissão da verdade ou incoerência na resposta apresentada.

O projeto foi devidamente submetido e protocolado na Plataforma Brasil, e apresenta parecer de número 2.906.052, sendo conduzido com base na Resolução n 466/2012 do Conselho Nacional de Saúde/Ministério da Saúde e a sua execução teve início somente após a aprovação pela referida plataforma.

Procedeu-se a análise estatística por meio do programa estatístico Statistical Package for Social Sciences (SPSS ${ }^{\circledR}$ ) versão 24. Além de estatísticas descritivas de frequência absoluta e relativa, média, desvio padrão e mediana, utilizou-se os testes inferenciais não paramétricos Qui-quadrado de Pearson ou teste exato de Fisher e o teste U de Mann-Whitney. Aceitouse como valor de significância estatística um $\mathrm{p}<0,05$. Os resultados foram apresentados em tabelas e gráficos e a discussão foi efetivada a partir da revisão de literatura, que serviu de sustentação científica, fundamentando a pesquisa.

\section{Resultados e Discussão}

A análise dos dados revelou que a média de idade entre as participantes da pesquisa é de 21,6 anos, que 92\% ( $\mathrm{n}=46$ ) delas são solteiras e 8\% (n=4) casadas. O estudo contou com a participação de mulheres com idade entre 17 e 34 anos, sendo que a maioria das pesquisadas $(84 \%, \mathrm{n}=42)$ apresentaram faixa etária entre 17 e 23 anos (Tabela 1).

Tabela 1 - Estado civil e idade de estudantes do sexo feminino da FSM - 2018

\begin{tabular}{|c|c|c|}
\hline & $\mathrm{N}$ & $\%$ \\
\hline \multicolumn{3}{|l|}{ Estado Civil } \\
\hline Solteira & 46 & 92,0 \\
\hline Casada & 4 & 8,0 \\
\hline \multicolumn{3}{|l|}{ Idade } \\
\hline Entre 17 e 23 anos & 42 & 84,0 \\
\hline Entre 23,1 a 29 anos & 5 & 10,0 \\
\hline Entre 29,1 a 34 anos & 3 & 6,0 \\
\hline
\end{tabular}

Fonte: Autores.

Resultados semelhantes quanto ao predomínio da faixa etária entre 17 e 23 anos foram encontrados na pesquisa de Felipe e colaboradores (2013), realizada com acadêmicas da Universidade Federal de Alfenas (UNIFAL - MG), que verificou predominância de mulheres com idade entre 17 e 20 anos, correspondendo a 48,3\% da amostra total.

Ainda com relação à caracterização sociodemográfica, o estudo de Brugge e colaboradores (2017), realizado com 42 universitárias da Universidade Estadual do Centro Oeste - Unicentro, no município de Guarapuava, Paraná, também revelou que maior parte das entrevistadas eram solteiras, representando $65,4 \%$ da sua amostra. No mesmo trabalho, a idade média observada foi de $25,29 \pm 6,56$ anos.

A maioria das participantes relatou não ter filhos $(94 \%, n=47)$. Quando questionadas se já tentaram engravidar, observou-se que $2 \%(\mathrm{n}=1)$ responderam que tentaram engravidar e não conseguiram, $90 \%(\mathrm{n}=45)$ nunca tentaram ou quiseram engravidar e $8 \%(n=4)$ relataram que já engravidaram (Tabela 2$)$. 
Tabela 2 - Gestação entre estudantes da FSM - 2018;

\begin{tabular}{ccc}
\hline & $\mathrm{N}$ & $\%$ \\
\hline Tem filhos & 3 & 6,0 \\
$\quad$ Sim & 47 & 94,0 \\
Não & & \\
Tentou engravidar e não conseguiu & 1 & 2,0 \\
Sim & 45 & 90,0 \\
Nunca tentou & 4 & 8,0 \\
Engravidou & & \\
\hline
\end{tabular}

Fonte: Autores.

O estudo de Felipe e colaboradores (2013) também encontrou um percentual parecido de universitárias que não possuíam filhos (90,7\%). Acredita-se que tal dado se justifique pelas dificuldades enfrentadas ao conciliar a maternidade, vida acadêmica, trabalho e atividades doméstica, além da idade média das participantes desta pesquisa e o fato de estarem solteiras.

A Tabela 3 demonstra que todas as participantes relataram ouvir falar da SOP e a maioria relatou não possuir casos na família $(62 \%, n=31)$. Entre as que disseram ter familiares com SOP, houve maioria de pessoas com parentesco de $4^{\circ}$ grau $(35,3 \%, \mathrm{n}=6)$.

Tabela 3 - Avaliação do conhecimento da SOP e observação de casos na família das estudantes da FSM - 2018

\begin{tabular}{ccc}
\hline & $\mathrm{N}$ & $\%$ \\
\hline Ouviu falar da SOP & 50 & 100,0 \\
Sim & 0 & 0,0 \\
Não & & 38,0 \\
Familiar com SOP & 19 & 62,0 \\
Sim & 31 & \\
Não & & 23,5 \\
Grau de parentesco do familiar* & 4 & 17,6 \\
I $^{\circ}$ grau & 3 & 23,5 \\
$2^{\text {o }}$ grau & 4 & 35,3 \\
$3^{\circ}$ grau & 6 & \\
$4^{\circ}$ grau & & \\
\hline
\end{tabular}

* participantes puderam assinalar mais de uma opção de resposta; por isso, o número de resposta é maior do que o número de participantes. Fonte: Autores.

O caráter familiar foi avaliado, pois a literatura sugere o componente familiar como uma das causas do surgimento da síndrome. Podem estar envolvidos em tal predisposição, variantes genômicas em genes relacionados à biossíntese, regulação e ação dos andrógenos, à ação e à secreção da insulina, à secreção e à ação das gonadotrofinas e à síntese e metabolismo do ácido retinóico, bem como genótipos pró-inflamatórios (Silva; Pardini; Kater, 2006).

Com relação às manifestações clínicas características da SOP, $4 \%(\mathrm{n}=2)$ das entrevistadas relataram não apresentar nenhum sintoma clínico da disfunção. Observou-se também, que 44\% ( $\mathrm{n}=22)$ apresentaram até 2 manifestações, 26\% ( $\mathrm{n}=13$ ) informaram possuir 3 sinais típicos da SOP, 20\% (n=10) apresentavam 4 características e $6 \%(\mathrm{n}=3)$ disseram apresentar 5 sintomas da síndrome (Tabela 4). 
Tabela 4 - Avaliação da presença de manifestações clínicas da SOP entre as estudantes da FSM - 2018

\begin{tabular}{ccc}
\hline & $\mathrm{N}$ & $\%$ \\
\hline Manifestações clínicas características da SOP* & & 4,0 \\
0 & 2 & 26,0 \\
1 & 13 & 18,0 \\
2 & 9 & 26,0 \\
3 & 13 & 20,0 \\
5 & 10 & 6,0
\end{tabular}

* participantes puderam assinalar mais de uma opção de resposta; por isso, o número de resposta é maior do que o número de participantes. Fonte: Autores.

Quanto à prevalência da síndrome dos ovários policísticos, o estudo revelou uma porcentagem de 24\% (n=12) das entrevistadas com o distúrbio. Além disso, $2 \%(\mathrm{n}=1)$ relatou ter diagnóstico inconcluso acerca da síndrome. Quando questionadas a respeito da conduta médica para diagnosticar a SOP, 46,2\% informaram a realização de exames de imagem (ultrassonografia), 30,8\% citaram o exame clínico (alterações do ciclo menstrual), ademais, a observação de sinais físicos de hiperandrogenismo (exame físico) representou $11,5 \%$ da conduta médica e exames laboratoriais também foram relatados por 11,5\% destas mulheres. Todas as participantes com SOP relataram fazer algum tipo de tratamento. A medida terapêutica mais empregada foi o uso de anticoncepcionais orais, seguido da adoção de um estilo de vida saudável. Nenhuma das entrevistadas fazia uso de medicamentos sensibilizantes à insulina (Tabela 5).

Tabela 5 - Prevalência da SOP entre acadêmicas da FSM e observação dos métodos diagnósticos e terapêuticos empregados 2018

\begin{tabular}{ccc}
\hline & $\mathrm{N}$ & $\%$ \\
\hline Tem SOP & & 24,0 \\
Sim & 12 & 74,0 \\
Diago & 37 & 2,0 \\
Diagnóstico Inconcluso & 1 & 30,8 \\
Clínico & & 11,5 \\
Físico & 8 & 46,2 \\
Imagem & 3 & 11,5 \\
Laboratorial & 12 & 100 \\
Sim & 3 & 0 \\
Não & & \\
Faz tratamto & 12 & 85,7 \\
Anticoncepcional & 0 & 14,3 \\
Estilo de vida saudável & & 0 \\
Medicamento sensibilizante à insulina & 12 & 2 \\
\end{tabular}

\footnotetext{
* participantes puderam assinalar mais de uma opção de resposta; por isso, o número de resposta é maior do que o número de participantes. Fonte: Autores.
}

Segundo Moreira e contribuintes (2013) a SOP é um distúrbio endócrino-ginecológico que afeta de $6 \%$ a $10 \%$ das mulheres em idade fértil. Já de acordo com Baptista, Vieira e Meireles (2018) a prevalência da SOP oscila entre 1,8\% a 15\% da população, sendo tal estatística influenciada pela etnia e critérios diagnósticos empregados. Rehme e colaboradores (2013b) por sua vez, determinaram que de $5 \%$ a $8 \%$ da população feminina em idade reprodutiva apresenta a síndrome. Facio-Lince 
García e auxiliares (2015) apresentou um percentual de 6\% a 21\% da disfunção. Deste modo, a literatura aponta percentuais de $1,8 \%$ até $21 \%$ de prevalência da SOP, estatística inferior à encontrada no presente estudo.

De acordo com o consenso de Rotterdam (2003), promovido pela European Society for Human Reproduction and Embriology (ESHRE) e pela American Society for Reproductive Medicine (ASRM) o diagnóstico de SOP deve ser dado a partir da constatação de no mínimo dois dos seguintes critérios: ciclos amenorreicos ou anovulação crônica, evidências clínicas ou laboratoriais de hipeandrogenismo e a presença de ovários com múltiplos cistos à ultrassonografia, desde que sejam excluídas outras alterações que provoquem sinais semelhantes, tais como a síndrome de Cushing, deficiência enzimática da suprarrenal, hiperprolactinemia, e distúrbios da glândula tireoide (Marcondes; Barcellos; Rocha, 2011). Por esta razão, as entrevistadas puderam assinalar mais de um método diagnóstico ou conduta médica.

O estudo em questão corrobora com o de Fernandes (2013), que diz que, apesar de pouco específica, a observação de ovários com múltiplos cistos na ultrassonografia da pelve é encontrado com grande frequência em pacientes com SOP, todavia pesquisas apontam que cerca de $20 \%$ de mulheres afetadas pela síndrome não apresentem ovários policísticos e ainda, aproximadamente $25 \%$ de mulheres normais e sadias exibam a característica em algum momento da vida.

No que se refere ao tratamento com anticoncepcional oral, resultados semelhantes foram encontrados na pesquisa de Brugge e colaboradores (2017), que identificou que $71,4 \%$ das participantes da sua pesquisa faziam uso de anticoncepcional oral sendo que $33,3 \%$ destas possuíam SOP.

Já o estudo de Felipe e colaboradores (2013), apontou que aproximadamente 49,3\% das participantes da sua pesquisa faziam uso da pílula, e destas, $12,9 \%$ destinava ao tratamento da síndrome dos ovários policísticos. O restante do percentual foi distribuído entre evitar a gravidez (31,8\%), seguido pelo desejo de regular o ciclo menstrual (26,4\%), controlar a tensão prémenstrual e cólica $(18,0 \%)$ e tratamento de acnes $(11,0 \%)$.

O uso de contraceptivos orais combinados (COC) é amplamente praticado, visto que apresenta grande eficácia no tratamento do hiperandrogenismo e irregularidades menstruais em pacientes com SOP. Os mesmos geralmente contem estrogénios e progesterona, que reduzem os níveis de andrógenos e inibem a proliferação do endométrio, respectivamente. Vale ressaltar que, a anovulação prolongada encontrada na síndrome leva a uma contínua estimulação endometrial pelos estrogénios, situação que implica na elevação de riscos relacionados ao surgimento de hiperplasia e neoplasia do endométrio (Baptista; Vieira; Meireles, 2016).

A adoção de estilo de vida saudável, que inclui a prática de exercícios físicos aliados a uma alimentação balanceada é a primeira linha de tratamento especialmente quando se trata de mulheres obesas com SOP. Nessas situações, a perda de peso, consequente de tais hábitos saudáveis ajuda na regulação do ciclo menstrual, diminuição da testosterona total e resistência à insulina e aumento da globulina ligadora de hormônios sexuais (SHBG) (Moura et al., 2011; Júnior et al., 2015).

Desde a década de 1990, os agentes sensibilizadores de insulina, principalmente a metformina (MTF), vêm sendo alvo de estudos e desde então observa-se considerável eficácia no reestabelecimento dos ciclos ovulatórios em pacientes com SOP, através do bloqueio da ação excessiva da insulina nas células da teca. No entanto, o tratamento com esse grupo de fármacos só é viável em casos onde a presença de resistência insulínica é comprovada, nos quais os benefícios de sua administração surgem de três a seis meses de tratamento (Cahill; O'brien, 2015).

A Figura 1 demostra as manifestações clínicas mais frequentes entre as mulheres com SOP entrevistadas. O sintoma clínico mais comum é a alteração do ciclo menstrual, que acomete cerca de $91,6 \%$ das portadoras da SOP deste estudo. O segundo sintoma mais frequente foi o excesso de oleosidade da pele, presente em $75 \%$ das mulheres com a disfunção, seguido de acne (66,6\%), alopécia (50\%), hirsutismo (33,3\%) e glicemia alterada ou diabetes $(8,3 \%)$. 
Figura 1 - Manifestações clínicas mais frequentes entre as mulheres com SOP da FSM - 2018

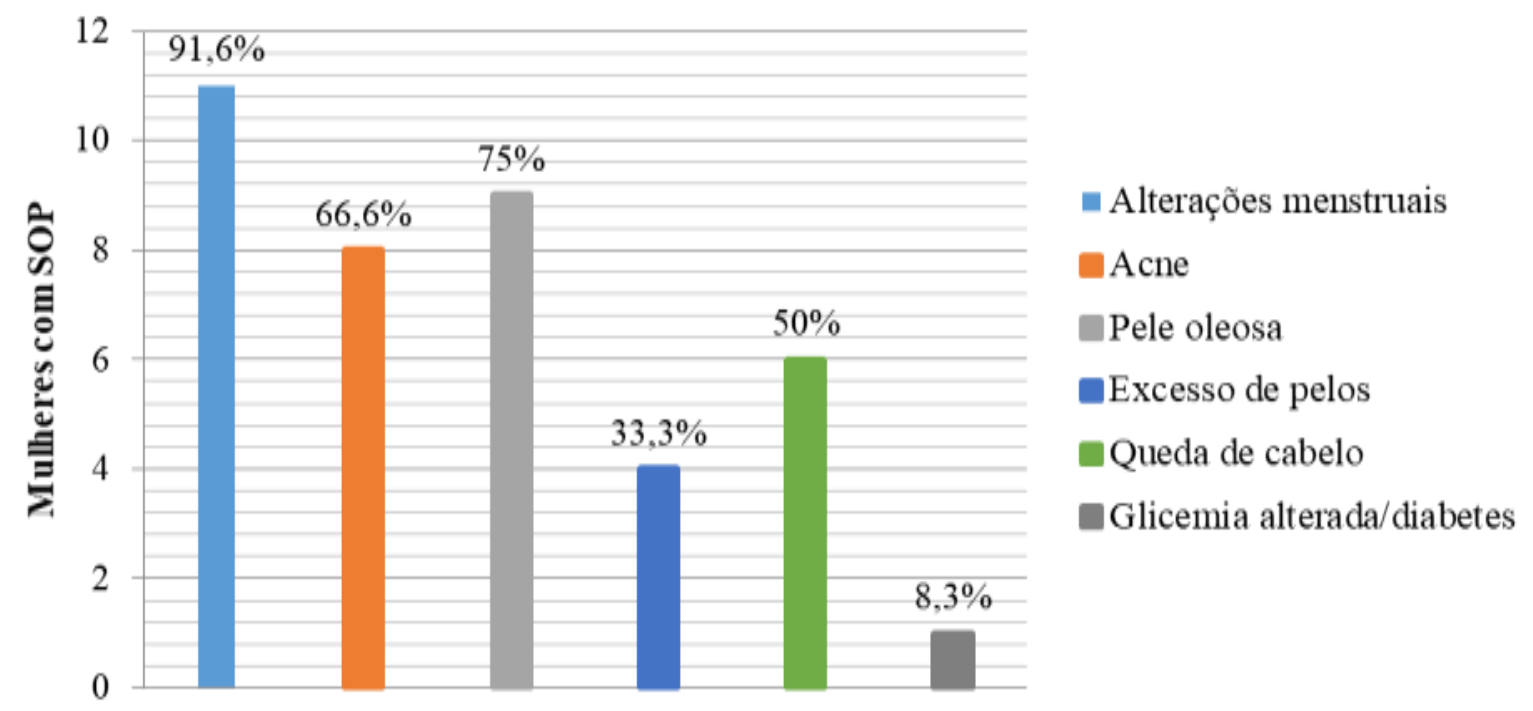

Fonte: Autores.

A pesquisa de Ávila e colaboradores (2014), também evidenciou que as alterações menstruais representam a irregularidade mais frequente entre as mulheres com SOP. Tal estudo revelou que $91 \%$ das entrevistadas tinham um ciclo menstrual desarmônico, e destas, $28 \%$ apresentavam oligomenorreia e $63 \%$ amenorreia. No mesmo trabalho foi observado ainda que o hirsutismo estava presente em $72 \%$ das entrevistadas e $49 \%$ das mesmas apresentavam acne.

Semelhantemente, Sousa e colaboradores (2013) relatou que em sua pesquisa, considerando os critérios diagnósticos de acordo com o Consenso de Rotterdam, a manifestação clínica mais frequente foi a irregularidade menstrual, relatada por 88,5\% das pacientes. Já o hiperandrogenismo clínico acometeu 68\% das mulheres com SOP, das quais 59\% apresentaram hirsutismo, 28,2\% acne e 9,0\% alopecia.

A pesquisa de Pontes e contribuintes (2012) que avaliou a presença de intolerância à glicose (IG) e diabetes mellitus do tipo $2(\mathrm{DM}$ - 2) em pacientes com SOP, através dos exames de glicemia em jejum e teste oral de tolerância a glicose (TOTG) constatou que 9,3\% destas mulheres apresentaram glicemia alterada, 10,1\% tinham IG e 5,7\% possuíam DM - 2. O presente estudo apontou resultados estatísticos semelhantes quanto a presença de glicemia alterada e diabetes em mulheres com SOP.

Vale destacar, que, observou-se ainda que entre as mulheres que declararam não possuir SOP ou ter diagnóstico inconcluso para a doença houve um considerado número de manifestações clínicas sugestivas da síndrome.

A Tabela 6 mostra que a média da idade das participantes da pesquisa que apresentavam a SOP foi de 20,5 anos e a das que não possuíam a disfunção foi de 22,03 anos. A mediana de idade de quem não possui SOP é maior, porém essa diferença não foi estatisticamente significativa $(\mathrm{p}=0,19)$. 
Tabela 6 - Comparação das medianas de idade entre as estudantes da FSM que possuem SOP e as que não possuem - 2018

\begin{tabular}{cccc}
\hline & \multicolumn{2}{c}{ Idade } \\
\hline Tem SOP & Média & Desvio padrão & Mediana \\
\hline Sim & 20,50 & 1,67 & 20,50 \\
Não & 22,03 & 3,84 & 21,00 \\
\hline$p$-valor & & & 0,19 \\
\hline
\end{tabular}

Fonte: Autores.

A Figura 2 mostra as médias de idade na comparação entre presença e ausência de SOP. Conforme pode ser visto, a média de idade de quem não possui SOP é maior, no entanto, considerando que existe sobreposição dos intervalos de confiança dos dois grupos, não se pode considerar que exista diferença estatisticamente significativa.

Figura 2 - Intervalos de confiança das médias de idade da prevalência de SOP entre estudantes da FSM - 2018

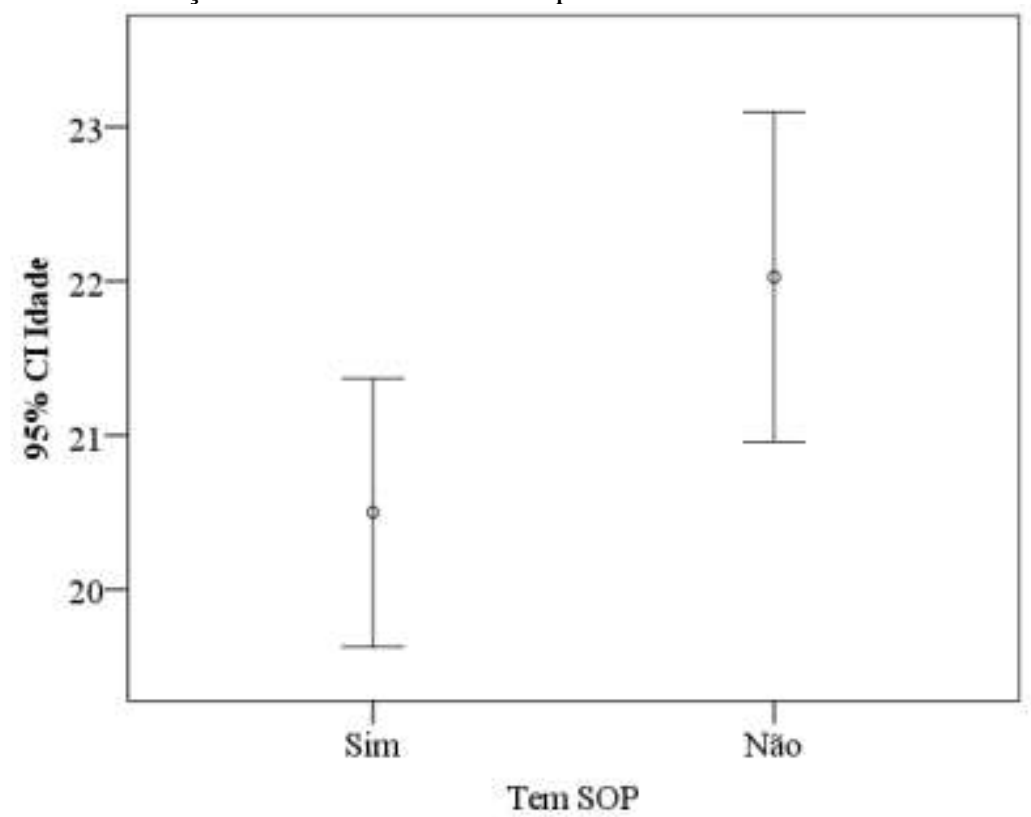

Fonte: Autores.

A Tabela 7 mostra que houve associação estatisticamente significativa entre familiar com SOP e ter a síndrome. Proporcionalmente, mais mulheres que relataram ter familiares acometidas pelo distúrbio tinham SOP.

Tabela 7 - Associação entre presença de SOP, SOP na família e filhos entre estudantes da FSM - 2018

\begin{tabular}{cccc}
\hline & \multicolumn{2}{c}{ Tem SOP } & p-valor \\
\cline { 2 - 3 } & Não & Sim & \\
Familiar com SOP & & & \\
Não & $26(86,7 \%)$ & $4(13,3 \%)$ & $0,04^{*}$ \\
Sim & $11(57,9 \%)$ & $8(42,1 \%)$ & \\
Tem filhos & & & 0,31 \\
Não & $34(73,9 \%)$ & $12(26,1 \%)$ & \\
\hline Sim & $3(100,0 \%)$ & $0(0,0 \%)$ &
\end{tabular}

* teste exato de Fisher. Fonte: Autores. 
Tal dado sugere a condição genética envolvida na etiopatogenia da síndrome dos ovários policísticos, que também pode ser influenciada por fatores ambientais. De acordo com Freitas e colaboradores (2017), tal herança genética é do tipo poligênica, onde os genes de biossíntese, ação e regulação de andrógenos e os que abrangem processo inflamatório crônico, aterosclerose e resistência insulínica, vem sendo os mais comumente relacionados à síndrome.

Sabe-se que cerca de $70 \%$ das mulheres com SOP apresentam problemas relacionados à fertilidade (Moreira et al., 2013). No entanto, acredita-se que, o dado do estudo em questão quanto a mulheres com SOP e filhos, que buscou avaliar a capacidade reprodutiva das mesmas, e demostrou que nenhuma das participantes com a síndrome tem filhos, pode se justificar pela idade média das universitárias, o percentual alto de mulheres que nunca tentaram engravidar e pelo fato de estarem solteiras.

\section{Considerações Finais}

Conclui-se que a síndrome dos ovários policísticos compreende um dos distúrbios endócrino-ginecológicos que mais acomete as mulheres em idade fértil. Em comparação com outros estudos na área, o presente trabalho detectou um percentual relevante de prevalência de mulheres com SOP na Faculdade Santa Maria, da cidade de Cajazeiras, Paraíba, identificando a síndrome em $24 \%$ da amostra analisada.

Verificou-se que os principais métodos utilizados para diagnosticar foram o exame clínico, através da constatação de alterações do ciclo menstrual, bem como o de imagem, por meio da ultrassonografia, que possibilita identificar os ovários com tamanho e forma anormais. Tais resultados demonstram a necessidade de realização de exames laboratoriais na grande maioria dos casos, afim de que se excluam outras causas de hiperandrogenismo, importante para que não haja um diagnóstico errôneo. Além disso, o anticoncepcional oral se apresentou como o tratamento mais utilizado pelas portadoras. Pudemos também confirmar nesta pesquisa a presença do fator genético atrelado à SOP, sugerido por diversos autores.

Apesar de representar um risco à saúde e bem-estar feminino, observou-se que há um déficit literário de dados epidemiológicos acerca da SOP. É de suma importância que novos estudos no âmbito populacional sejam realizados com esta temática, explorando a prevalência da SOP em diferentes etnias e regiões, já que a mesma se configura como um importante determinador do bem-estar feminino. Recomenda-se a realização de pesquisas com tamanhos amostrais maiores e intervalo de idade que atinja diferentes fases da vida da mulher, buscando analisar questões que não puderam ser exploradas neste trabalho, como as relacionadas à infertilidade da mulher com SOP.

\section{Referências}

Ávila, M. A. P. de et al. Acantose nigricante: inter-relações metabólicas inerentes à síndrome dos ovários policísticos. Revista Brasileira de Ginecologia e Obstetrícia, 36(9), 410-415, 2014.

Baptista, D., et al. Síndrome do Ovário Poliquístico na adolescência. Nascer e Crescer, 25(4), 227-235.

Brugge, F. A., et al. Associação entre diagnóstico de síndrome de ovários policísticos, estado nutricional e consumo alimentar em mulheres em idade fértil. RBONE-Revista Brasileira de Obesidade, Nutrição e Emagrecimento, 11(62), 117-124.

Cahill, David J., O'brien, Katherine. Polycystic ovary syndrome (PCOS): metformin. BMJ clinical evidence, 2015.

Facio-Lince G. et al. Síndrome de ovario poliquístico y complicaciones metabólicas: más allá del exceso de andrógenos. Revista chilena de obstetricia y ginecología, 80(6), 515-519.

Felipe, T. B., et al. Avaliação do conhecimento sobre os contraceptivos orais entre as universitárias http://dx. doi. org/10.5892/ru vrv. 2013.111. 5867. Revista da Universidade Vale do Rio Verde, 11(1), 58-67, 2013.

Fernandes, L. G., et al. Síndrome dos ovários policísticos: Uma abordagem epidemiológica. 2013. Tese (Doutorado em saúde pública). Universidade Federal da Bahia, Salvador, 2013.

Freitas, L. R. A., et al. Uso de metformina em mulheres obesas com Síndrome do Ovário Policístico. Revista de Ciências Médicas, $25(2), .87-97$. 
Research, Society and Development, v. 10, n. 4, e58310414412, 2021

(CC BY 4.0) | ISSN 2525-3409 | DOI: http://dx.doi.org/10.33448/rsd-v10i4.14412

Kogure, G. S., \& Reis, R. M. dos. Progressive Resistance Training as Complementary Therapy for Polycystic Ovarian Syndrome. Revista Brasileira de Ginecologia e Obstetrícia, 39(6), 255-257.

Marcondes, J. A. M., et al. Dificuldades e armadilhas no diagnóstico da síndrome dos ovários policísticos. Arquivos Brasileiros de Endocrinologia \& Metabologia, 55(1), 6-15, 2011.

Moreira, S. da N. T., et al. Qualidade de vida e aspectos psicossociais da síndrome dos ovários policísticos: um estudo quali-quantitativo. Rev Bras Ginecol Obstet, 35(11), 503-510.

Moura, H. H. G., et al. Síndrome do ovário policístico: abordagem dermatológica. Anais Brasileiros de Dermatologia, 2011.

Organização Mundial Da Saúde. Mulheres e saúde: evidências de hoje, agenda de amanhã. 2011.

Pontes, A. G., et al. A importância do teste de tolerância à glicose oral no diagnóstico da intolerância à glicose e diabetes mellitus do tipo 2 em mulheres com síndrome dos ovários policísticos. Revista Brasileira de Ginecologia e Obstetrícia, 128-132.

Rehme, M. F. B. et al. Manifestações clínicas, bioquímicas, ultrassonográficas e metabólicas da síndrome dos ovários policísticos em adolescentes. Revista Brasileira de Ginecologia e Obstetrícia, 35(6), 249-254.

Rehme, M. F. B., et al. Contribuição do hiperandrogenismo para o desenvolvimento de síndrome metabólica em mulheres obesas com síndrome dos ovários policísticos. Revista Brasileira de Ginecologia e Obstetrícia, 562-568.

Rojas, J. M. P. Síndrome de Ovarios Poliquísticos. Asociación Costarricense de Medicina Legal y Disciplinas Afines, 35.

Silva, R. do C., et al. Síndrome dos ovários policísticos, síndrome metabólica, risco cardiovascular e o papel dos agentes sensibilizadores da insulina. Arquivos Brasileiros de Endocrinologia \& Metabologia, 50(2), 281-290.

Soares Júnior, J. M., et al. Polycystic ovary syndrome: controversies and challenges. Revista da Associação Médica Brasileira, 61(6), 485-487.

Spritzer, P. M., et al. Neoplasias associadas à síndrome dos ovários policísticos. Arquivos brasileiros de endocrinologia \& metabologia= Brazilian archives of endocrinology and metabolism. 49(5), 805-810. 